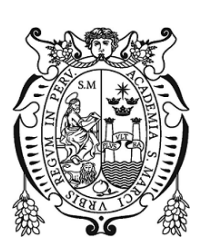

Revista de Investigación de Física 23(2), (Jul-Dic 2020)

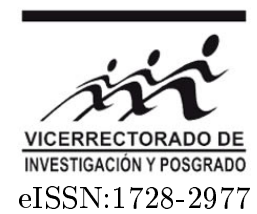

\title{
Evaluación de la operación de un medidor remoto de calidad del aire basado en sensores de lectura directa
}

\author{
Oscar Baltuano ${ }^{* 1,2}$, Fernando Rosas ${ }^{2}$, Renzo $_{\text {Chan }}{ }^{1}$, Octavio Fashé ${ }^{2}$ y Eduardo Montoya ${ }^{2}$ \\ ${ }^{1}$ Instituto Peruano de Energía Nuclear, Lima, Perú \\ ${ }^{2}$ Universidad Nacional Mayor de San Marcos, Lima, Perú
}

Recibido 28 marzo 2020 - Aceptado 10 mayo 2020

\begin{abstract}
Resumen
Se reporta la evaluación de un instrumento de bajo costo, desarrollado por los autores, para la medición continua y autónoma de las concentraciones de $\mathrm{NO}_{2}, \mathrm{SO}_{2}, \mathrm{NO}, \mathrm{H}_{2} \mathrm{~S}, \mathrm{CO}, \mathrm{PM} 2.5$ y PM10 en una zonas urbanas, el mismo que ha sido puesto a prueba en un punto ubicado en el distrito de Bellavista, provincia del Callao, Lima, Perú. Se presentan los resultados obtenidos para un lapso de tres meses de observación. Dichos resultados son consistentes con los valores esperados para una zona de escaso tránsito vehicular y por ello de baja contaminación.

Palabras clave: calidad del aire, gases contaminantes, bajo costo, medición remota.

\section{Evaluation of the operation of a remote air quality meter based on direct reading sensors}

Abstract

The evaluation of a low cost instrument, developed by the authors, for the continuous measurement of the chemical concentrations of $\mathrm{NO}_{2}, \mathrm{SO}_{2}, \mathrm{NO}, \mathrm{H}_{2} \mathrm{~S}, \mathrm{CO}, \mathrm{PM} 2.5$ and PM10 in urban zones, it has been tested at a point located in the Bellavista district, Callao province, Lima, Peru. The results obtained for an observation time of three months are report. These results are consistent with the expected values for a zone of low vehicle transit and then of low contamination.
\end{abstract}

Keywords: air quality, polluting gases, low cost, remote measurement.

\section{Introducción}

La calidad del aire, entendida como la cuantificación de la concentración de ciertos gases tales como $\mathrm{NO}_{2}, \mathrm{SO}_{2}$, $\mathrm{NO}, \mathrm{H}_{2} \mathrm{~S}, \mathrm{CO}, \mathrm{O}_{3}$ entre otros, y la concentración de material particulado suspendido en aire, es una de las principales preocupaciones en entornos urbanos debido a los efectos dañinos que tienen para la salud humana o el medio ambiente según estudio de la Defensoría del Pueblo del año 2006 [Def06].

Generalmente, la medición de la concentración de gases contaminantes en aire, requiere de técnicas de laboratorio complejas y difíciles de implementar como métodos automáticos de medición en línea o en tiempo real. Estas técnicas, aceptadas como métodos de referencia, incluyen por ejemplo, fluorescencia UV para $\mathrm{SO}_{2}$ y $\mathrm{H}_{2} \mathrm{~S}$, infrarrojo no dispersivo para $\mathrm{CO}$, quimioluminiscencia para $\mathrm{NO}_{2}$, entre otros que han sido establecidos por la Dirección General de Salud Ambiental (2005) [Dgs05].
Sin embargo, en la actualidad, gracias al desarrollo de la instrumentación y micro-electrónica, se disponen de dispositivos de medición electroquímicos que cuentan con características de operación muy ventajosas, tales como: respuesta de lectura directa, tamaño compacto, bajo costo, facilidad de integración, portabilidad, selectividad y baja complejidad de implementación. Como ejemplo, la compañía Alphasense (2013) [Alp13] fabrica sensores electroquímicos de gases selectivos compactos (Figura 1), que pueden ser fácilmente integrados dentro de equipos y sistemas de medición automáticos similares al propuesto por Mead (2013) [Mea13].

De forma similar, para la determinación de la concentración de material particulado menor a 2,5 micras y menor a 10 micras en aire, cuya medición de forma convencional utiliza métodos gravimétricos, ahora se cuenta con dispositivos muy sensibles que utilizan técnicas ópticas de conteo de partículas suspendidas a un cos-

\footnotetext{
*obaltuano@ipen.gob.pe
} 
to considerablemente menores y con mayor facilidad de automatización e integración como son el Nova SDS011 (2014) [Nov14] y el DFRobot SEN0177 (2015) [Dfr15].

Por otro lado, la miniaturización de estos dispositivos ha abierto otras posibilidades de uso, tales como su integración en redes amplias de sensores o incluso redes colaborativas de medición usando por ejemplo, las redes de telefonía celular, lo que amplía considerablemente la cobertura de medición y proporciona mayor cantidad de información como lo demuestran Al-Ali (2010) [Ali10], Carvalho (2009) [Car09] y Hasenfratz (2012) [Has12].

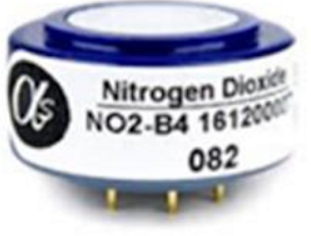

(A)

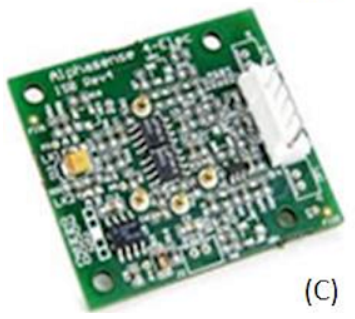

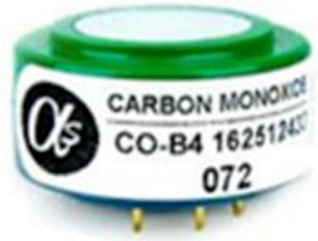

(B)

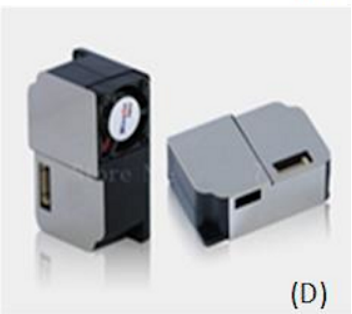

Figura 1: (A) y (B) Dos sensores selectivos de gases de la serie B4 de Alphasense y (C) su correspondiente tarjeta de acondicionamiento electrónico (ISB). (D) Un contador de partículas por dispersión de luz.

A nivel local, no se han hecho desarrollos importantes utilizando estos sensores de lectura directa. Los sistemas en línea utilizados por las entidades competententes como el Ministerio del Ambiente poseen pocos puntos de muestreo y al ser equipos de marcas comerciales generan una dependencia tecnológica y económica que en ocasiones imposibilita la operatividad de los mismos. En grandes ciudades es cada vez más frecuente recurrir a redes colaborativas para ampliar la cantidad de datos que permitan hacer mejores correlaciones entre los niveles de calidad de aire y su impacto en la población como lo sugiere Khedo (2010) [Khe10]. Desarrollos como el mostrado en este trabajo permiten contribuir al establecimiento de este tipo de redes.

\section{Experimental}

\section{Equipamiento}

El equipo de medición de calidad del aire que ha sido evaluado (Figura 2) fue diseñado y construido en el marco del proyecto 161-FINCyT-IA-2013 y tiene la capacidad para determinar la concentración de los siguientes gases contaminantes: $\mathrm{NO}_{2}, \mathrm{NO} \mathrm{SO}_{2}, \mathrm{NO}, \mathrm{H}_{2} \mathrm{~S}, \mathrm{CO}, \mathrm{O}_{3}$. Además ha sido equipado con un medidor de material particulado que permite la determinación de los niveles de concentración PM10 y PM2.5. Este es un contador de partículas por dispersión láser de bajo costo DFRobot SEN0177 (2015) [Dfr15]. Este equipo es totalmente autónomo, no requiriendo la intervención de ningún operador. Únicamente es necesario contar con una toma de energía eléctrica. El núcleo de la operación del equipo es una tarjeta Arduino DUE (2015) [Ard15] equipada con dos convertidores ADC LTC1867 de 16 bits (2008) [Lin08] . Estos dispositivos permiten digitalizar la señal analógica que proporcionan los sensores de gases contaminantes. Con la finalidad de reducir al mínimo la contribución de ruido electrónico se realiza un promediado de 10,000 muestras, a una tasa de muestreo de 200,000 muestras por segundo, para obtener un valor de medición. Cada sensor selectivo de gases requiere la medición del voltaje del electrodo de trabajo y del electrodo auxiliar, por lo cual se realizan 12 mediciones en total ( 2 por cada uno de los 6 sensores de gases). En el caso de los sensores de material particulado, temperatura y humedad, esto no es necesario debido a que estos dispositivos son de tipo digital y proporcionan los datos ya procesados.

El equipo cuenta con 4 modos para almacenar y/o transmitir la información colectada: almacenamiento de una memoria SD interna, transmisión de datos a una PC mediante una conexión USB, transmisión mediante una conexión Wi-Fi y transmisión de datos a través de una conexión de telefonía celular GSM/GPRS. En todos los casos la información es la misma y se almacena o transmite cada 5 segundos.

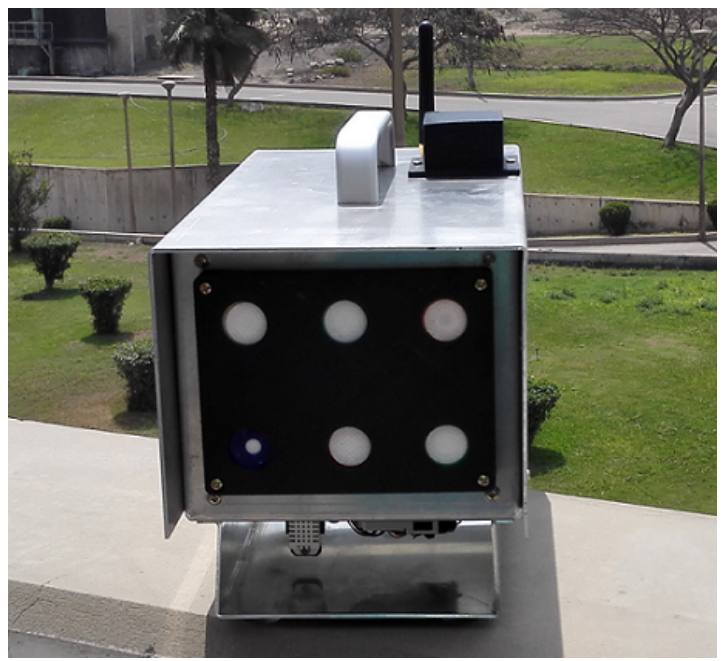

Figura 2: Vista frontal del medidor de calidad del aire utilizado. En primer plano se observan las entradas de cada sensor de gases contaminantes. En la parte inferior se encuentra montado el sensor de material particulado y el sensor de temperatura y humedad. 


\section{Calibración}

Para la utilización de los sensores electroquímicos, Alphasense recomienda su uso en conjunto con sus respectivas tarjetas Alphasense ISB (Individual Sensor Board) [Alp13] las cuales pueden ser calibradas en fábrica. Dichas tarjetas proporcionan una señal analógica de algunos mV que es utilizada para calcular la concentración de cada gas. Para realizar el cálculo de la concentración se debe tener en cuenta los valores de calibración del voltaje de compensación u offset de cada electrodo (WE y AE) de cada sensor y la sensibilidad (expresada en $\mathrm{nA} / \mathrm{ppm}$ ). Todos estos valores son proporcionados por el fabricante en el empaque de cada sensor, el mismo que viene ensamblado con la tarjeta ISB. De acuerdo a las especificaciones del fabricante, la sensibilidad define la relación entre el valor de voltaje medido y la concentración del gas contaminante. Dicho valor no cambia y se considera constante durante el periodo de vida del sensor. Los valores de offset de los electrodos representan una desviación que puede ir variando dependiendo del uso y de las condiciones de operación de los sensores. Teniendo en cuenta lo anterior, se realizó la determinación experimental de los valores de offset de cada electrodo para cada sensor utilizado en tanto se consideró la sensibilidad reportada en el empaque de cada sensor. Para ellos se acopló en cada sensor un dispositivo que permitiera la circulación de aire sintético en ausencia de cualquier gas contaminante (Figura 3). Cada sensor fue sometido a esta prueba durante 6 horas continuas y se tomó como valor de offset de cada electrodo el voltaje medido al finalizar dichas pruebas.

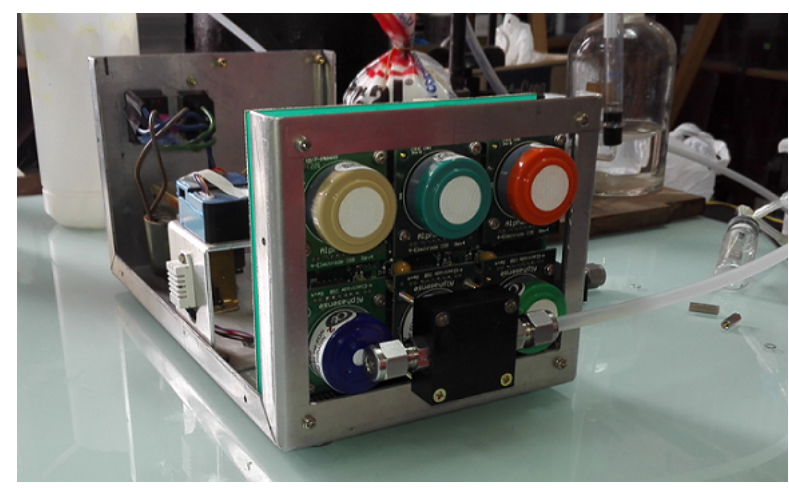

Figura 3: Configuración para determinación de valor de offset de cada sensor mediante la aplicación de aire sintético

Los resultados obtenidos fueron diferentes a los marcados en cada empaque, probablemente por el tiempo transcurrido desde su adquisición. Dichos valores se resumen en la Tabla 1 .

\begin{tabular}{ccccc}
\hline Gas & $\begin{array}{c}\text { WE Zero } \\
\mathrm{mV}\end{array}$ & $\begin{array}{c}\text { AE Zero } \\
\mathrm{mV}\end{array}$ & $\begin{array}{c}\text { Sensib. } \\
\mathrm{nA} / \mathrm{ppm}\end{array}$ & $\begin{array}{c}\text { Gain } \\
\mathrm{mV} / \mathrm{nA}\end{array}$ \\
\hline $\mathrm{CO}$ & 320 & 327 & 477 & 0.8 \\
$\mathrm{H} S$ & 284 & 304 & 1775 & 0.8 \\
$\mathrm{NO}$ & 285 & 278 & 735 & 0.8 \\
$\mathrm{NO}_{2}$ & 253 & 232 & -454 & -0.726 \\
$\mathrm{O}_{3}$ & 224 & 286 & -567 & -0.726 \\
$\mathrm{SO}_{2}$ & 343 & 350 & 370 & 0.8 \\
\hline
\end{tabular}

Tabla 1: Valores de offset de los electrodos de trabajo y auxiliar obtenidos mediante la aplicación de aire sintético.

La determinación de la concentración de cada uno de los gases medidos se realizó utilizando el procedimiento descrito en la hoja de especificaciones de Alphasense (2013) [Alp13] . El sensor de material particulado utilizado DFRobot SEN0177 no requiere ningún procedimiento de calibración por cuanto dicho sensor viene calibrado de fábrica y proporciona directamente los valores de concentración de PM10 y PM2.5 a través del microcontrolador integrado dentro de su tarjeta electrónica.

\section{Valores máximos permitidos}

Los valores obtenidos con el equipo de medición han sido contrastados con los límites máximos permitidos para cada contaminante. Dichos valores, de acuerdo a las normas vigentes, se resumen en la Tabla 2.

\begin{tabular}{|c|c|c|c|c|}
\hline \multirow[b]{2}{*}{ Gas } & \multirow[b]{2}{*}{$\Delta \mathrm{T}$} & \multicolumn{2}{|c|}{ Límite } & \multirow{2}{*}{$\begin{array}{c}\text { Referencia } \\
\text { D.S. }\end{array}$} \\
\hline & & $u g / m^{3}$ & $\mathrm{ppm}$ & \\
\hline \multirow{2}{*}{$\mathrm{SO}_{2}$} & Año & 80 & 30.5 & 074-2001-PCM \\
\hline & $24 \mathrm{~h}$ & 20 & 7.6 & 003-2008-MINAM \\
\hline \multirow{2}{*}{$C O$} & $8 \mathrm{~h}$ & 10000 & 8729 & \multirow{2}{*}{ 074-2001-PCM } \\
\hline & $1 \mathrm{~h}$ & 30000 & 26187 & \\
\hline \multirow{2}{*}{$\mathrm{NO}_{2}$} & Año & 100 & 53.2 & \multirow{2}{*}{ 074-2001-PCM } \\
\hline & $1 \mathrm{~h}$ & 200 & 106.3 & \\
\hline $\mathrm{O}_{3}$ & $8 \mathrm{~h}$ & 120 & 61.1 & 074-2001-PCM \\
\hline $\mathrm{H}_{2} \mathrm{~S}$ & $24 \mathrm{~h}$ & 150 & 76.4 & 003-2008-MINAM \\
\hline \multirow{2}{*}{$P M 10$} & Año & 50 & \multirow{2}{*}{ N.A. } & \multirow{2}{*}{ 074-2001-PCM } \\
\hline & $24 \mathrm{~h}$ & 150 & & \\
\hline \multirow{2}{*}{$P M 2.5$} & Año & 15 & \multirow{2}{*}{ N.A. } & \multirow{2}{*}{ 074-2001-PCM } \\
\hline & $24 \mathrm{~h}$ & 25 & & \\
\hline
\end{tabular}

Tabla 2: Límites máximos permitidos según los Estándares de Calidad Ambiental (ECA's) vigentes en el Perú. No se ha encontrado legislación vigente sobre los límites máximos para monóxido de nitrógeno

\section{Emplazamiento}

El medidor remoto fue instalado dentro del edificio de la Casa de la Juventud de la Municipalidad de Bellavista ubicado en la Calle Los Heros \# 200 en la Municipalidad Provincial del Callao (Figura 4). El equipo estuvo 
instalado a una altura de 5 metros dentro de una abertura sobre frente del edificio la misma que cuenta con una estructura de aluminio para ventilación que permite fácilmente el paso de aire hacia el interior. Esta ubicación se encuentra en la Calle Los Heros con coordenadas: latitud sur $12^{\circ} 03^{\prime} 41.2 z$ longitud oeste $77^{\circ} 07^{\prime} 50.3^{\prime \prime}$ (Figura 5). Se escogió la misma por tener una circulación libre del aire desde la parte exterior del edificio, además de contar con toma eléctrica para la operación del mismo y con la finalidad de reducir posibles daños por condiciones climáticas adversas o hurto.

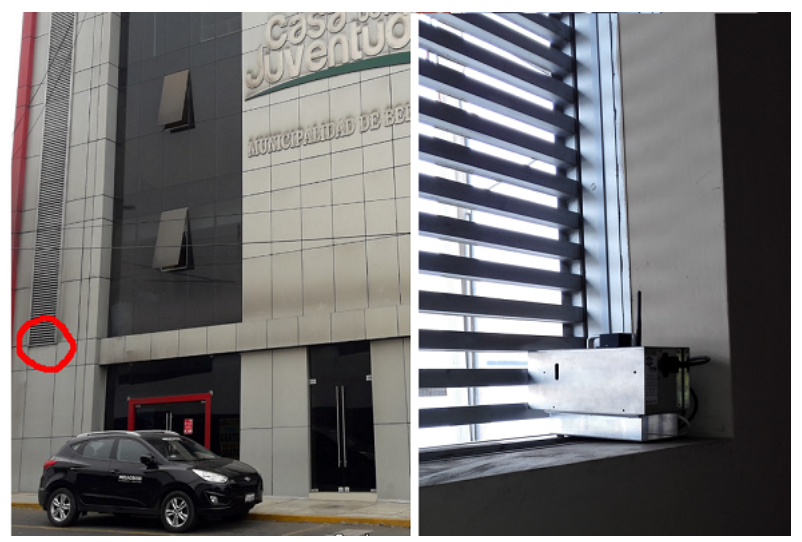

Figura 4: A la izquierda, frontis del lugar de emplazamiento del medidor en la calle Los Heros. El círculo en rojo indica la posición en la rejilla de ventilación donde fue instalado el medidor. A la derecha, vista interior del medidor instalado.

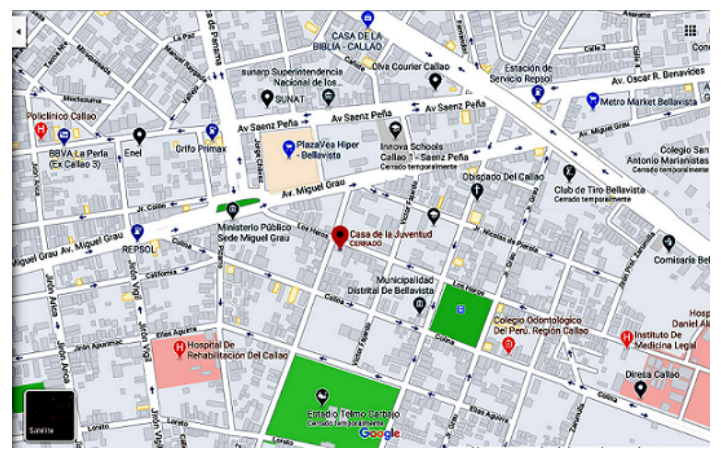

Figura 5: Mapa de la ubicación del punto de medición en la Casa de la Juventud (Marca color rojo).

\section{Resultados y Discusión}

Los datos obtenidos con el equipo de medición han sido recuperados desde la memoria interna SD y procesados posteriormente para calcular las concentraciones de cada gas contaminante según Alphasense (2013) [Alp13] . En el caso de material particulado, el sensor proporciona directamente el valor de concentración según DFRobot (2015) [Dfr15]. Las mediciones se realizaron en el periodo comprendido entre el 9 de febrero y el 31 de abril del año 2018. Las Figuras 6, 7 y 8 muestran las variaciones en la concentración de gases contaminantes y material particulado en dicho periodo considerando promedios cada 24 horas, lo que proporciona un valor por día. De acuerdo a los Estándares de Calidad Ambiental (ECA's) se recomiendan obtener valores de concentración promedio durante periodos de 24 horas y 8 horas.

Durante el periodo de adquisición de lecturas el equipo ha operado ininterrumpidamente de acuerdo a lo previsto en sus especificaciones. En el caso de los sensores de gases, no se ha observado un desplazamiento significativo del valor medio en el periodo de medición, esto significa que al menos durante el periodo de 3 meses no es necesario realizar ningún ajuste o re-calibración de los valores de offset de los electrodos de trabajo y auxiliar de cada sensor.

En la Figura 8 de las variaciones diarias en la concentración de material particulado se observa claramente la disminución de dicha concentración en días que corresponden con los fines de semana o feriados en los cuales la circulación de vehículos disminuye considerablemente. Este mismo comportamiento, aunque en mucha menor magnitud también se observa en las concentraciones diarias de gases.

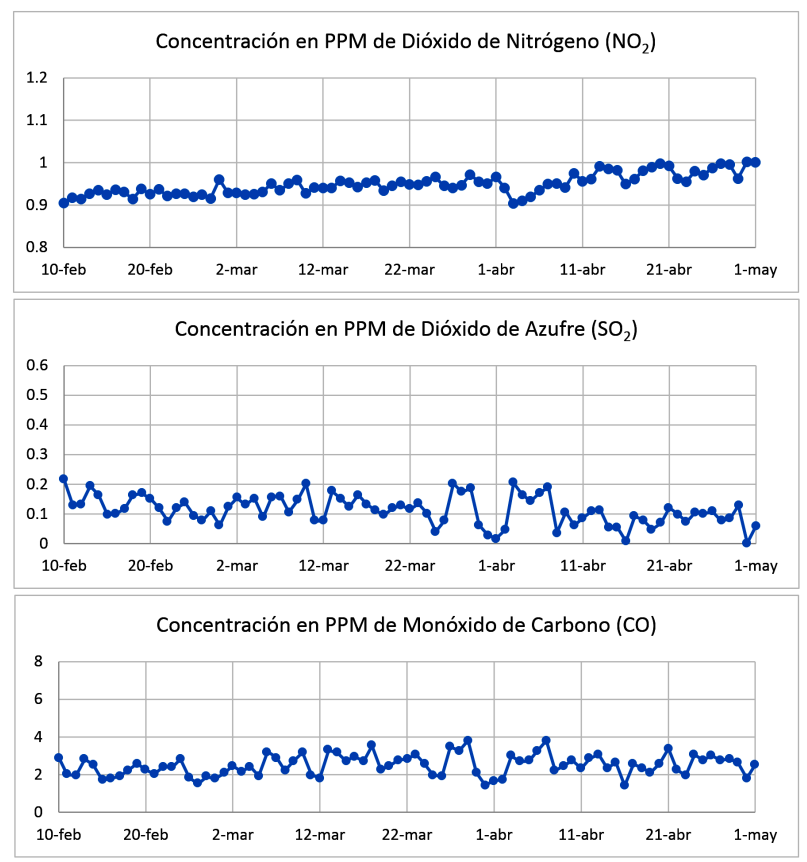

Figura 6: Concentración medida en $\mathrm{PPM}$ de $\mathrm{NO}_{2}, \mathrm{SO}_{2}$ y CO entre el 09 de febrero y el 31 de abril de 2018

Es posible obtener mayor cantidad de datos y una mejor correlación, poniendo en operación el medidor durante periodos más largos de tiempo y habilitando el acceso de datos automáticamente en forma remota, a través de 
un servidor en línea. Aunque se disponen de dos equipos de medición adicionales, los mismos deben pasar por el proceso de medición de los valores de offset de los electrodos de cada sensor.

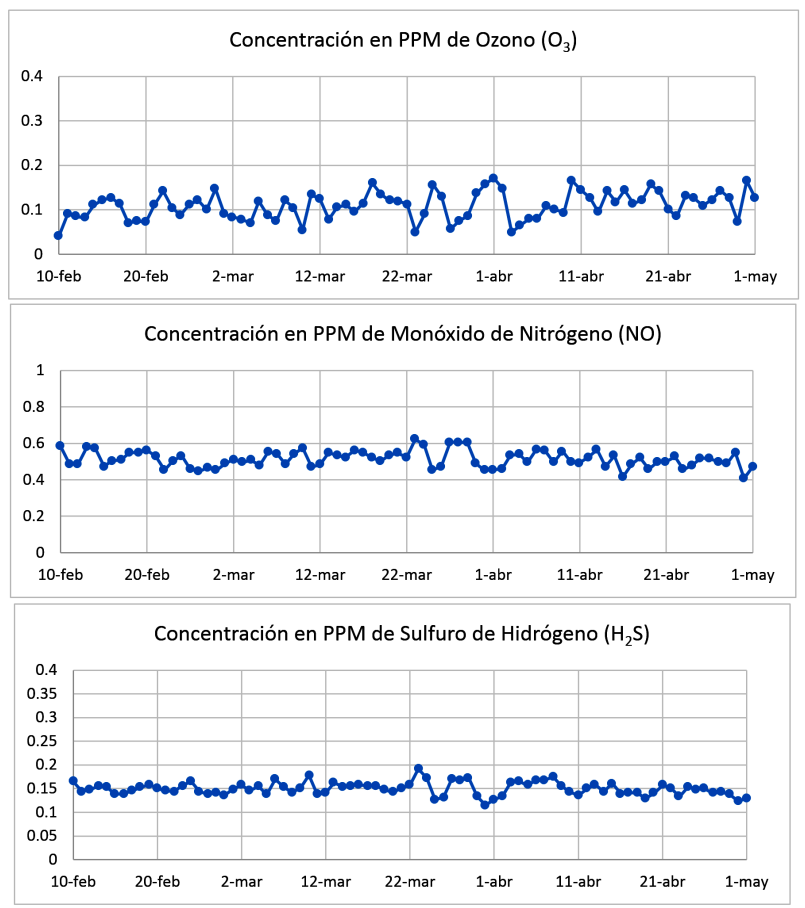

Figura 7: Concentración medida en PPM de $\mathrm{O}_{3}$, NO y $\mathrm{H}_{2} \mathrm{~S}$ entre el 09 de febrero y el 31 de abril de 2018

\section{Conclusiones}

Los resultados de las mediciones son consistentes con los valores esperados y muestran que la instrumentación descrita y evaluada en este trabajo es confiable y adecuada para el propósito de monitoreo de la calidad del aire en zonas urbanas. Los valores obtenidos, ya sea en promedios de 24 horas o de 8 horas en todos los casos, se han encontrado por debajo de los valores máximos permitidos según la legislación vigente. La variaciones en las concentraciones, en donde ha sido posible identificarlas, se deben fundamentalmente a variaciones en la temperatura ambiental lo cual está dentro de lo esperado si se tiene en cuenta que el lugar de emplazamiento tiene una baja circulación de vehículos motorizados los cuales constituyen la principal fuente de contaminación tanto en gases como en material particulado. Este primer prototipo está en condiciones de contribuir a la implementación de una red amplia con mayor cantidad de puntos de medición respecto de las redes actualmente disponibles con la consecuente reducción de nuestra dependencia tecnológica y la optimización de los recursos asignados este tipo de redes, facilitando la posibilidad de obtener mayor cantidad de datos en tiempo real y con ello permitir hacer mejores correlaciones entre la calidad del aire y los estudios epidemiológicos en la población.

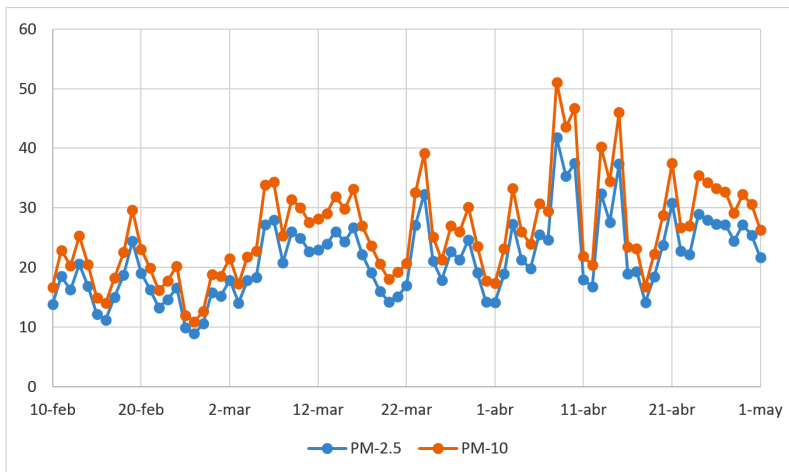

Figura 8: Concentración medida en $\mu \mathrm{gg} / \mathrm{m}^{3}$ de PM2.5 y PM10 entre el 09 de febrero y el 31 de abril de 2018

\section{Agradecimientos}

Este trabajo ha sido desarrollado gracias al Proyecto 161-FINCyT-IA-2013 "Diseño e implementación de un red piloto de sensores para monitoreo en tiempo real de parámetros de calidad del aire en la ciudad de Lima" el mismo que contó financiamiento monetario otorgado por Innóvate Perú para la ejecución del mismo. También participaron como entidades asociadas: la Universidad Nacional Mayor de San Marcos y la empresa GNR Integradores SAC.

\section{Referencias}

[Ali10] Al-Ali, A., Zualkernan, I. and Aloul, F. (2010). $A$ Mobile GPRS-Sensors Array for Air Pollution Monitoring, IEEE Sensors Journal, Vol. 10, No. 10, October 2010

[Alp13] Alphasense Ltd (2013). Alphasense 4-Electrode
Individual Sensor Board (ISB) User Manual. 0852217.

[Ard15] Arduino C.C. (2015). Getting started with the Arduino Due, web: http://arduino.cc/en/ pmwiki .php?n=Guide/ArduinoDue

[Car09] Carvalho, V., Lopes, J., Ramos, H. and Corrêa, F. (2009). City-Wide Mobile Air Quality Measure- 
ment System. IEEE Sensors 2009 Conference, Portugal.

[Def06] Defensoría del Pueblo (2006). La Calidad del Aire en Lima y su Impacto en la Salud y la vida de sus Habitantes. Informe Defensorial \# 116, web: https: //sinia.minam.gob.pe/download/file/fid/39237

[Dfr15] DFRobot Wiki (2015). PM2.5 Laser dust Sensor SKU SEN0177, web: https://wiki.dfrobot.com/ PM2.5_laser_dust_sensor_SKU_SEN0177

[Dgs05] Dirección General de Salud Ambiental (2005), Protocolo de Monitoreo de la Calidad del Aire y Gestión de los Datos. Perú, web: http://www.digesa.minsa.gob.pe/norma_ consulta/Protocolo-de-Calidad-del-Aire.pdf

[Has12] Hasenfratz, D., Saukh, O., Sturzenegger, S. and Thiele, L. (2012). Participatory Air Pollution Monitoring Using Smartphones. 2nd International Workshop on Mobile Sensing, April 16-20, 2012, Beijing, China
[Khe10] Khedo, K., Perseedoss, R. and Mungur, A. (2010). A Wireless Sensor Network Air Pollution Monitoring System. International Journal of Wireless \& Mobile Networks (IJWMN), Vol. 2, No 2, May 2010

[Lin08] Linear Technology Corporation (2008). LTC1863/LTC1867 12-/16-bit, 8-Channel, 200 ksps ADC's Datasheet, web: http: //www. linear.com/docs/2141/

[Mea13] Mead, M., Popoola, O., Stewart, G., Landshoff, P., Calleja, M., Hayes, M., Baldovi, J., MacLeod, M., Hodgson, T., Dicks, J., Lewis, A., Cohen, j., Baron, R., Saffell, J. and Jones, R. (2013). The use of electrochemical sensors for monitoring urban air quality in low-cost, high-density networks, Atmospheric Environment $70.186-203$.

[Nov14] Nova Fitness Co. Ltd (2014). Laser PM2.5 Sensor specification, Product model: SDS011, Version: V1.0 